\title{
Identification et stratégie d'élimination des allergènes
}

Oléagineux, Corps Gras, Lipides. Volume 9, Numéro 2, 112-4, Mars - Juin 2002, La filière

Auteur(s) : Gabriel PELTRE, Ecole supérieure de physique et chimie industrielle (ESPCI), LECA, Allergie et environnement, 10, rue Vauquelin, 75005 Paris, France.

Résumé : L'allergie touche au moins trois grands systèmes physiologiques de notre organisme. Le premier est le système immunitaire, qui produit des anticorps de la classe lgE à la suite de l'exposition à un allergène. Ce sont ces anticorps qui vont nous permettre d'identifier et de suivre à la trace ces allergènes. Une ré-exposition de l'organisme aux mêmes allergènes, touchant le système inflammatoire, va entraîner la libération de médiateurs, en particulier l'histamine, et induire les symptômes de l'allergie. Les effets du système nerveux central sur les deux autres systèmes ne seront pas abordés ici.

\section{ARTICLE}

Après un rappel des caractéristiques des allergènes, cet exposé rapportera les dispositions actuelles d'évaluation du risque allergénique, les compléments de méthodes attendus dans les prochaines années, les points à surveiller particulièrement, les bénéfices prévisibles de l'ingénierie génétique appliqué aux OGM en allergologie.

\section{Caractéristiques générales des trophallergènes}

Les allergènes sont une variété particulière d'antigènes, substances capables de susciter une réponse immunitaire dite " allergique ", liée à la synthèse d'IgE spécifiques. Les allergènes alimentaires, ou trophallergènes, en représentent un sous-groupe fort important. On distingue, dans les aliments, des milliers de protéines, dont seulement quelques-unes sont allergéniques $[5,6]$. On appelle allergène majeur un antigène purifié contre lequel au moins $50 \%$ des patients testés présentent des IgE spécifiques, et qui donne des tests cutanés immédiatement positifs, à une concentration très faible, chez au moins $90 \%$ des sujets ayant la maladie allergique en relation avec cet allergène. Les isoallergènes sont des molécules de même poids moléculaire et de fonctions biologiques identiques. Ils présentent une homologie d'identité des séquences amino-acides d'au moins $67 \%$. Ils se différencient par le point isoélectrique, qui dépend des charges positives et négatives des acides aminés.

Les protéines allergéniques ont subi des modifications post-transcriptionnelles : glycosylation, acylation, méthylation, etc. Celles-ci sont essentielles pour la structure tertiaire, dont peuvent dépendre des variations d'allergénicité. Nombre d'allergènes alimentaires ont un poids moléculaire moyen : entre 10000 et 70000 daltons. Quelques allergènes connus ont des tailles plus importantes, comme Ara h 1 (63,5 kd) et Ara h 2 (17 kd) qui existent sous forme de polymères de 200 à $300 \mathrm{kd}$. On peut penser qu'ils sont fragmentés lors de la digestion [7]. La plupart des allergènes alimentaires sont des glycoprotéines à point isoélectrique acide. Mais il existe des allergènes non glycosylés, et d'autre 
part, les déterminants carbohydrates ne paraissent pas jouer un rôle net dans l'allergie clinique. Ces glycoprotéines appartiennent à la famille des albumines (solubles dans l'eau) ou des globulines (solubles en soluté salin). Plus rarement, telles les gliadines, elles sont solubles dans l'alcool.

L'allergénicité est le fait de portions limitées de la protéine, les déterminants antigéniques ou épiscopes (figure 1). Ceux qui interagissent avec les lymphocytes $T$ sont appelés épitopes $T$, ceux qui se lient aux IgE, et qui interagissent avec les lymphocytes B, sont les épitopes B.

Un épitope est un petit peptide. Huit à neuf acides aminés constituent un épitope $T$, base de l'immunogénicité. Les épitopes $B$ ont une taille de 8 à 16 amino-acides [6]. Des épitopes réduits à cinq acides aminés ont toutefois été observés pour la farine de blé [8]. On distingue des épitopes séquentiels dépendant de l'enchaînement des acides aminés (structure primaire) et des épitopes conformationnels dépendant de la structure tertiaire ou quaternaire. Ceux-ci sont continus s'ils correspondent à un enchaînement d'acides aminés, ou discontinus (rapprochement spatial de séquences non contiguës). Généralement les épitopes réagissant avec les lymphocytes $T$ sont linéaires. Les épitopes réagissant avec les lymphocytes $B$ sont le plus souvent situés à la surface extérieure de la molécule : ils sont conformationnels, continus ou discontinus [6]. Mais il existe des exceptions : les protéines du lait de vache possèdent des épitopes $B$ linéaires, enterrés dans des parties hydrophobes des molécules et qui ne deviennent accessibles aux IgE qu'après la protéolyse [9]. Les épitopes conformationnels sont plus fragiles que les épitopes séquentiels. II existe là aussi des exceptions : certains épitopes d'Ara $\mathrm{h} 1$, se liant aux IgE spécifiques, sont situés aux extrémités d'assemblage des monomères en trimère Ara $\mathrm{h} 1$; bien que dépendant de cette structure quaternaire, ils sont très résistants $[7,10,11]$. Des épitopes particuliers sont représentés par des résidus carbohydrates, greffés sur le squelette protéique des glycoprotéines, abondantes dans les pollens, fruits et légumes. On considère actuellement qu'ils ont peu ou pas d'implication dans l'allergie clinique [12]. La connaissance de la structure primaire, et des épitopes linéaires de nombreux allergènes alimentaires n'a pas permis de dégager des caractéristiques communes d'allergénicité. Des particularités conformationnelles de la surface extérieure des molécules sont plausibles. Peu de structures tridimensionnelles sont connues. Elles semblent indiquer que le trait commun des allergènes serait leur forme sphérique. Un autre élément notable est la fréquence de l'activité enzymatique de ces allergènes [13]. En ce qui concerne les allergènes végétaux, ils font partie de familles définies par leurs fonctions, très conservées à travers l'évolution génétique : les pan-allergènes. Leurs homologies expliquent la réactivité croisée observée entre des familles botaniques taxonomiquement bien différentes [14].

La résistance à la dénaturation thermique caractérise certains épitopes, dits thermostables. Les protéines de lait de vache (caséines et alpha-lactalbumine encore plus que la bêta-lactoglobuline et la sérum-albumine bovine), gardent leur pouvoir de liaison aux IgE spécifiques après chauffage à 80$100{ }^{\circ} \mathrm{C}$ pendant 15 minutes [6]. Inversement, l'alpha-lactalbumine et la bêta-lactoglobuline de lait de jument ne sont plus reconnues par les IgE spécifiques après $25 \mathrm{mn}$ d'ébullition, la dénaturation de I'alpha-lactalbumine étant déjà très avancée au bout de $10 \mathrm{~min}$ [6]. Les allergènes de poisson, de crevette, l'ovalbumine et l'ovomucoïde sont très résistants à la chaleur $[6,15]$. Les allergènes majeurs de l'arachide sont particulièrement thermostables à $100^{\circ} \mathrm{C}$ [7]. Les allergènes de riz et de soja ne sont que partiellement dénaturés par chauffage de $60^{\circ} \mathrm{C}$ à $80^{\circ} \mathrm{C}$ pendant une demi-heure à 
une heure [16]. Les deux types d'allergènes, thermostables et thermolabiles, coexistent dans un même aliment. Selon le profil individuel de sensibilisation, cela explique que certains allergiques tolèrent un aliment cuit, à l'inverse d'autres sujets.

La résistance à la protéolyse est une caractéristique de l'allergénicité alimentaire. Dans un modèle de digestion artificielle, on vérifie la stabilité, pendant une heure, de l'allergénicité de l'inhibiteur de Künitz (soja) d'une sub-unité de bêta-conglycine (soja), de Ara h 2 (arachide), de Bra j 1 et Sin a 1 (moutarde). Un nouveau pan-allergène, la famille des protéines de transport lipidique, se révèle très résistant également [17]. À l'opposé, différentes protéines enzymatiques, qui ne sont pas allergéniques, du soja, du blé, de la feuille d'épinard, de l'orge, de la pomme de terre, sont complètement détruites en moins d'une minute $[10,18,19]$. Ara $\mathrm{h} 1$ est fragile, perdant son immuno-réactivité en 15 secondes [19]. Les albumines animales (viandes) ont un comportement intermédiaire [20]. Toutefois, de petits peptides restent encore allergéniques, comme le montrent les allergies alimentaires dues à des hydrolysats poussés de protéines de lait [21]. Les épitopes conformationnels sont plus sensibles à la chaleur et à la protéolyse que les épitopes linéaires. La résistance à un $\mathrm{pH}$ modérément acide caractérise nombre de trophallergènes. Un allergène majeur de l'arachide ainsi que l'ovalbumine ne sont pas dénaturés à pH 3 [6].

\section{Évaluation du risque allergénique d'un OGM}

Différentes questions doivent être envisagées :

1) Si le gène vient d'un végétal allergisant connu, la protéine transgénique n'est-elle pas un allergène mineur?

2) Une modification du métabolisme de cette protéine dans le végétal-hôte ne peut-elle conduire à l'expression d'une quantité importante d'un allergène mineur comme cela a été observé dans le cas de l'expression de l'albumine $2 \mathrm{~S}$ de la noix du Brésil (NB) dans le soja ? (en sus, bien entendu, du fait que cette albumine était l'allergène majeur de la NB) [3].

3) Sa présence peut-elle modifier l'allergénicité des protéines de la plante-hôte ?

4) Si l'origine du gène est une plante sans potentialité allergénique connue, présente-t-elle une réactivité croisée avec les allergènes connus (peut-elle se lier à des IgE spécifiques d'un allergène) ?

5) Dans les deux éventualités, est-elle douée d'immunogénicité (risque de sensibilisation de novo) ?

Dispositions actuelles d'évaluation du risque allergénique d'un OGM

Mises en place en 1996 par un groupe de travail de l'IFBC (International Food Biotechnology Council) et I'ILSI (International Life Sciences Institute), ces dispositions ont été revues et complétées par un comité d'experts FAO/WHO, dont les recommandations ont été publiées en janvier 2001 [12, 22]. Leur présentation a été faite antérieurement $[18,19,23]$. Rappelons que la première étape est la recherche d'une homologie de séquences avec les allergènes répertoriés dans les banques de données accessibles sur Internet (Gen Bank, Swiss Prot, PIR, EMBL). On tenait compte en 1996 d'une séquence de huit acides aminés contigus identiques. En 2001, on prête attention à l'identité de six acides aminés contigus, ou bien à $35 \%$ d'identité sur l'ensemble des acides aminés. Cette étape est 
limitée par le nombre actuel d'allergènes identifiés, et par le repérage d'épitopes potentiels de type linéaire. Or les réactions croisées sont dues à des homologies d'épitopes $B$, dont beaucoup sont conformationnels, liés à la structure tertiaire des allergènes qui est encore peu connue [24, 25]. Si le gène provient d'un végétal à potentiel allergénique connu, il est nécessaire de rechercher un risque de liaison aux IgE spécifiques de sérums de sujets allergiques à cette plante. La nécessité de disposer d'un maximum de sérums de patients allergiques à l'espèce végétale dont est issu le gène d'intérêt ressort des évaluations de probabilité de détection d'un allergène majeur ou mineur : il a été calculé que 14 sérums sont suffisants pour prédire que cette protéine n'est pas un allergène majeur, avec une probabilité de 99,9\% (six sérums assurent une probabilité de détection de $95 \%$ ). La détection d'un allergène mineur correspondant à $20 \%$ de sera spécifiques, nécessite 24 sérums pour assurer une probabilité de $99 \%$ et 17 sérums pour une probabilité de détection de $95 \%$ [12]. On voit à quel point il est important de disposer d'un nombre suffisant de sérums validés par des moyens diagnostiques standardisés comportant en particulier des tests de provocation orale en double insu. Si le gène provient d'un végétal sans potentiel allergénique connu, il est préconisé sous l'égide de la $\mathrm{FAO} / \mathrm{WHO}$, de rechercher une éventuelle réactivité croisée avec toutes les familles ou sortes d'allergènes existants, en testant la protéine vis-à-vis de six séries de sérums contenant des IgE spécifiques de monocotylédones (pollens de graminées, riz), de dicotylédones (pollens d'arbres, d'herbacées, céleri, arachide, fruits secs, oléagineux, latex, etc.), du groupe de moisissures (Alternaria, Aspergillus, Cladosporium, trichophyton, levures), du groupe invertébrés (sérums spécifiques d'acariens, blattes, chironomides, crevette, soie), du groupe vertébrés (sérums spécifiques d'épithelia animaux et de protéines sériques des mêmes sources, de lait, de poisson, d'œuf). Dans chaque groupe, il est recommandé la mise à disposition de 25 sérums spécifiques de pneumallergènes et de 25 sérums spécifiques d'allergènes alimentaires. Cette recommandation n'a pas l'assentiment d'auteurs américains car ils mettent en doute la bonne caractérisation des sérums nécessaires qui dépendraient de banques de sérums commerciales [2]. Cette objection a une grande valeur mais l'obstacle n'est pas dirimant, si le soin de ces banques est confié à la responsabilité de I'Agence française de sécurité sanitaire des aliments (Afssa). Il lui reviendra de créer les relations nécessaires avec des centres d'allergologie alimentaire, hospitalo-universitaires de référence.

Les protéines transgéniques non cross réactives seront ensuite étudiées dans des modèles de digestion artificielle, puisque la rapide destruction de leur antigénicité paraît caractériser les protéines végétales non allergéniques. Au bout d'un temps d'incubation avec la pepsine allant jusqu'à 1 heure, la présence résiduelle de fragments de plus de 3,5 kDa fait considérer une allergénicité potentielle. Les modèles de digestion artificielle n'approchent pas encore la complexité de la réalité. En particulier, le système artificiel de digestion intestinale a été peu appliqué. Or certaines protéines se sont révélées très résistantes aux enzymes intestinales, conduisant à l'interrogation sur leur dégradabilité in vivo, dans des cas d'achylie, ou agastrie chirurgicale, ou vidange accélérée lors de gastro-entérites. Ces trois étapes - recherche d'homologie de séquences, de liaison à des IgE spécifiques, de dégradabilité par la digestion - ne permettent pas de répondre à la question d'une allergénicité accrue de l'OGM, non plus qu'à celle d 'une nouvelle immunogénicité. II est donc nécessaire d'envisager le développement de modèles animaux qui, idéalement, devraient être aptes à une réponse allergique, similaire à la gradation observée chez I'homme, selon que le potentiel allergénique est fort (cacahuète), moyen (lait), ou presque nul (épinard). Ils devraient disposer d'un système immunologique intestinal proche du GALT. Leur réponse immunologique devrait s'adresser aux mêmes épitopes [26, 27]. Certains proposent le chien [28], d'autres le rat 
Sprague-Dawley [29], le rat Brown Norway [30], certaines souches de souris comme C3H/HeJ [27, 31], le cobaye, le porcelet, etc.

À l'intérieur d'une même espèce, comme la souris, d'importantes différences de réponse immunologique peuvent être observées [32]. Une étude a concerné l'immunogénicité comparée d'un soja naturel et d'un soja génétiquement modifié, sans relever de différence [29].

\section{Réflexions sur les risques des OGM}

Nombre de réflexions naissent dans ce nouveau domaine, dont la première, préliminaire, doit dénoncer la fausse sécurité engendrée par le faible taux de protéine exprimée qui, pour les OGM résistant aux herbicides, représente effectivement moins de $0,4 \%$ des protéines totales. Cependant, l'allergène majeur de la pomme ne représente que de 0,3 à $1,5 \%$ des protéines. De même, l'allergie alimentaire à la pêche est bien connue : or ce fruit ne contient que $0,05 \%$ de protéines.

D'autre part, la création d'OGM à visée nutritionnelle conduira à des taux de protéines transférées beaucoup plus importants. II faut recommander aux industriels la transparence quant à la quantité présente de protéine transgénique, afin de calculer la quantité habituellement consommable. Celleci sera comparée avec les doses minimales réactives des allergènes connus, qui, pour l'arachide sous forme de graine, sont de l'ordre de quelques centaines de microgrammes [33]. II faudra prêter attention aux OGM à base de graines comportant une sur-expression d'enzymes, car il est bien connu que de nombreux allergènes (et auto-antigènes) sont des protéines enzymatiques.

À ces deux égards, les huiles transgéniques, provenant de graines surexprimant quantitativement et qualitativement leurs acides gras, devront être particulièrement surveillées. De multiples enzymes, à des taux non précisés, sont utilisés [34]. Or le risque allergique des huiles est démontré pour l'arachide, le tournesol, le soja. On est frappé par la minime quantité des allergènes susceptible de provoquer la réaction allergique (de l'ordre de quelques dizaines de microgrammes de protéines) alors qu'il faut une quantité cent fois supérieure de protéines sous forme de graines pour déclencher une réaction chez les mêmes individus. Les modifications de métabolisme de la protéine transgénique sont déjà connues. Dans le cas de l'albumine $2 S$ de la noix du Brésil, son métabolisme dans le soja conduisait à une accumulation d'un précurseur qui s'est révélé être un allergène mineur. L'albumine d'Amaranthe dans la pomme de terre transgénique subit une importante glycosylation, le PM passant de 30 à $50 \mathrm{kDa}$ [35]. II conviendra de vérifier l'absence de répercussion sur les protéines propres de l'hôte [36]. Ces questions indiquent que les études d'immunogénicité sur les modèles animaux devront s'adresser non seulement à la protéine transgénique isolée, mais encore à l'organisme génétiquement modifié dans sa totalité. Cela paraît d'autant plus important que les végétaux hôtes seront principalement le soja, le riz, le maïs, le blé peut-être, la pomme de terre, caractérisés par l'universalité de leur consommation, et dont l'allergénicité spécifique est bien connue. II sera encore nécessaire de surveiller le risque de modifications imprévues des protéines transgéniques du fait des technologies alimentaires qui leur seront ultérieurement appliquées. Les modifications de structures par repliements moléculaires pourraient faire apparaître des motifs conformationnels homologues d'épitopes B existants. Enfin, il faudra veiller à ce que des protéines transgéniques dévolues à des aliments pour bétail ne soient pas frauduleusement utilisées pour l'alimentation humaine : à cet égard, un lupin transgénique (Lupinus angustifolius), porteur d'une 
albumine de tournesol, pour le bétail, pourrait présenter un risque pour l'homme, car cette albumine est un allergène des graines de tournesol [37].

Le cas du maïs Starlink a confirmé que le risque de contamination involontaire est également à prendre en considération puisque la protéine cry9c (figure 2) autorisée pour le maïs transgénique servant à l'alimentation du bétail, avait bien des caractéristiques d'allergène potentiel : glycoprotéine de poids moléculaire de $68 \mathrm{kDa}$, résistance relative à la dégradation protéolytique, à la chaleur et au traitement acide, et surtout inductrice d'IgE spécifiques chez le rat Norway [38].

La surveillance de ces risques devra comporter une surveillance post-commercialisation nécessitant la mise en place d'une structure d'allergo-vigilance dont le principe a déjà été détaillé [23]. II est notable que le rapport FAO WHO de janvier 2001 intègre cette recommandation. Cette surveillance s'adressera en fait au cadre plus large des aliments nouveaux. Cette surveillance devra comporter une allergo-vigilance des cliniciens, mais également les techniques appropriées d'étude sur les sérums suspects d'allergie. Le dépistage d'IgE spécifiques aux protéines transgéniques devrait utiliser non seulement la protéine recombinante (isolée de l'espèce vectrice), mais également la protéine isolée de l'aliment transgénique, et ce afin d'être certain d'analyser la responsabilité éventuelle d'épitopes conformationnels dépendant de la glycosylation de la protéine dans l'espèce végétale haute, comme le fait remarquer Kuiper.

Les bénéfices attendus des OGM en allergologie

La première catégorie d'OGM a eu pour but d'accroître le rendement en douant les OGM de propriétés insecticides, de résistance aux herbicides, etc. La seconde vague s'oriente vers l'acquisition de qualités nutritionnelles accrues. La troisième phase pourrait s'intéresser à des aliments ayant des propriétés fonctionnelles et thérapeutiques, que l'on pourrait nommer alicaments. Les premiers essais chez l'homme, de pomme de terre ou de laitue transgéniques exprimant l'antigène HBS et la sous-unité B de l'entérotoxine d'Escherichia coli, ont montré qu'ils permettraient une vaccination orale, surtout applicable aux affections diarrhéiques. Toutefois, les résultats dépendent, pour l'antigène HBS, de la coexistence d'un adjuvant (toxine cholétique) et l'entérotoxine est elle-même un adjuvant, si bien que la question que de tels produits favorisent une sensibilisation à l'aliment lui-même doit être posée [39]. On peut faire I'hypothèse que des alicaments trouveraient une application dans les maladies allergiques qui sont actuellement au cinquième rang de fréquence des maladies. L'élaboration d'aliments hypoallergéniques a été réalisée pour le riz, grâce à l'introduction d'un gène anti-sens [40]. Le taux de l'allergène majeur a été diminué de $60 \%$. Un effet thérapeutique a été observé chez des sujets allergiques au riz, porteurs de dermatite atopique [41]. Une voie explorée est la création, par mutagenèse dirigée, de variétés d'arachide hypoallergéniques, exprimant une forme modifiée de deux allergènes majeurs Ara $h 2$ et Ara $\mathrm{h} 3$, à faible pouvoir de liaison aux IgE spécifiques [42, 43]. L'insertion, dans une protéine propre de la plante-hôte, de peptides à activité biologique, ouvrira d'autres horizons [44]. L'insertion d'épitopes $T$ vaccinants d'allergènes majeurs [45], I'insertion de peptides cytokiniques ou de molécules adjuvantes orientant la réponse immunitaire vers une activité Th1 préférentielle [46] ou immunorégulatrices comme TGF-beta [47], sont encore une vue de l'esprit, mais de telles recherches contribueraient grandement à une acceptabilité des OGM par les populations. 


\section{REFERENCES}

1. PRIDMORE R, CROUZILLAT D, et al. (2000). Genomics, molecular genetics and the food industry. J Biotechnol, $78: 251-8$.

2. TAYLOR S, HEFLE S (2001). Will genetically modified foods be allergenic? J Allergy Clin Immunol, $107: 765-71$.

3. NORDLEE J, TAYLOR S, et al. (1996). Identification of a Brazil nut allergen in transgenic soybeans. New Eng J Med, $334:$ 688-92.

4. KANNY G, MONERET-VAUTRIN DA, et al. (2001). Population study of food allergy in France. J Allergy Clin Immunol, $108:$ 133-40.

5. BUSH R, HEFLE S (1996). Food allergens. Crit Rev Food Sci Nutr, 36 (S) : 119-63.

6. TAYLOR SL, LEHRER SB (1996). Principles and characteristics of food allergens. Crit Rev Food Sci Nutr, 36 : S91-118.

7. BURKS W, SAMPSON H, BANNON G (1998). Peanut allergens. Allergy, 53 : 725-30.

8. TANABE S, ARAI S, YANAGIHARA Y, et al. (1996). A major wheat allergen has a Gln-Gln-Gln-Pro-Pro motif identified as an IgE-binding epitope. Biochem Biophys Res Communications, 219 : 290-3.

9. WAL J (2001). Structure and function of milk allergens. Allergy, $56:$ 35-8.

10. BURKS A, WILLIAMS L, THRESHER W, et al. (1992). Allergenicity of peanut and soybean extracts altered by chemical or thermal denaturation in patients with atopic dermatitis and positive food challenges. J Allergy Clin Immunol, 90 : 889-97.

11. MALEKI S, KOPPER R, SHIN D, et al. (2000). Structure of the major peanut allergen Arah 1 may protect IgEbinding epitopes from degradation. J Immunol, 164 : 5844-9.

12. FAO/WHO (2001). Joint FAO/WHO Expert Consultation on allergenicity of foods derived from biotechnology.

13. BREDEHORST R, DAVID K (2001). What establishes a protein as an allergen? J Chromatogr $B$ Biomed Sci Appl, 756 : 33-40.

14. BREITENEDER H, EBNER C (2000). Molecular and biochemical classification of plant-derived food allergens. J Allergy Clin Immunol, 106 : 27-36.

15. HEFLE S (1999). Impact of processing on food allergens. In : Jackson et al. Impact of Processing on Food Safety. New York : Kluwer Academic/ Plenum : 107-19.

16. URISU A, YAMADA K, MASUDA S, et al. (1991). 16-kilodalton rice protein is one of the major allergens in rice grain extract and responsible for cross-allergenicity between cereal grains in the Poaceae family. Int Arch Allergy Appl Immunol, 96 : 244-52. 
17. ASERO R, MISTRELLO G, RONCAROLO D, et al. (2001). Lipid transfer protein: a pan-allergen in plant-derived foods that is highly resistant to pepsine digestion. Int Arch Allergy Immunol, 124 : 67-9.

18. ASTWOOD J, FUCHS R (1996). Preventing food allergy : Emerging technologies. Trends Food Sci Technol, $7: 219-26$.

19. ASTWOOD J, FUCHS RL (1996). Allergenicity of foods derived from transgenic plants. In : Wüthrich B, Ortolani C. Highlights in food allergy. Basel : Karger, 32 : 105-20.

20. FIOCCHI A, RESTANI P, RIVA E, et al. (1998). Heat treatment modifies the allergenicity of beef and bovine serum albumin. Allergy, $53: 798-802$.

21. KELSO J, SAMPSON H (1993). Food protein-induced enterocolitis to casein hydrolysate formulas. J Allergy Clin Immunol, 92 : 909-10.

22. METCALFE DD, ASTWOOD JD, TOWNSEND R, SAMPSON HA, TAYLOR SL, FUCHS RL (1996). Assessment of the allergenic potential of foods derived from genetically engineered crop plants. Crit Rev Food Sci Nutr, 36 : 165-86.

23. MONERET-VAUTRIN DA (2000). Évaluation du risque allergique des aliments transgéniques d'origine végétale. CR Acad Agric Fr, 86 : 77-85.

24. WAL JM (1997). Évaluation de l'innocuité des aliments issus d'organismes génétiquement modifiés. Rev fr Allergol, 37 : 326-33.

25. WAL JM, PASCAL G (1998). Benefits and limits of different approaches for assessing the allergenic potential of novel foods. Allergy, $53:$ 98-101.

26. BUCHANAN B (2001). Genetic engineering and the allergy issue. Plant Physiol, 126 : 5-7.

27. LI XM, SEREBRISKY D, LEE SY, et al. (2000). A murine model of peanut anaphylaxis: T and B cell responses to a major peanut allergen mimic human responses. J Allergy Clin Immunol, 106 : 150-8.

28. ERMEL R, KOCK M, et al. (1997). The atopic dog: a model for food allergy. Lab Anim Sci, 47 : 40- 9.

29. CHANG H, BAE Y, et al. (2001). Allergenicity test of genetically modified soybean in Sprague Dawley Rats. Arch Pharm Res, 24 : 256-61.

30. MILLER K, MEREDITH C, SELO I, WAL JM (1999). Allergy to bovine betalactoglobulin: specificity of immunoglobulin E generated in the Brown Norway rat to tryptic and synthetic peptides. Clin Exp Allergy, 29 : 1696-704.

31. DEARMAN R, KIMBER I (2001). Determination of protein allergenicity: studies in mice. Toxicol Lett, 120 : 181-6.

33. HUANG C, SAMPSON H, et al. (1999). Strain dependent protection fromallergic reactions by peanut allergen plasmid-DNA immunization in mice. J Allergy Clin Immunol, 103 : S 238.

33. TAYLOR S, HEFLE S, BINDSLEV-JENSEN C, et al. (2001). Factors affecting the determination of threshhold doses for allergenic foods: how much is too much? J Allergy Clin Immunol (accepted). 
34. HAZEBROEK J (2000). Analysis of genetically modified oils. Progress in Lipid Research 39 : 477 506.

35. CHAKRABORTY S, CHAKRABORTY N, DATTA A (2000). Increased nutritive value of transgenic potato by expressing a nonallergenic seed albumin gene from Amaranthus hypochondriacus. Proc Natl Acad Sci, 97 : 3724-9.

36. BURKS AW, FUCHS RL (1995). Assessment of the endogenous allergens in glyphosate-tolerant and commercial soybean varieties. J Allergy Clin Immunol, 96 : 1008-10.

37. MOLVIG L, TABE LM, EGGUM BO, et al. (1997). Enhanced methionine levels and increased nutritive value of seeds of transgenic lupins (Lupinus angustifolius L.) expressing a sunflower seed albumin gene. Proc Natl Acad Sci, 94 : 8393-8.

38. KUIPER HA, KLETER GA, NOTEBORN HPJM, KOK EJ (2001). Assessment of the food safety issues related to genetically modified foods. The Plant Journal 27 : 503-28.

39. MATOBA N, DOYAMA N, et al. (2001). Design and production of genetically modified soybean protein with anti-hypertensive activity by incorporating potent analogue of ovokinin. FEBS Letters, $497: 50-4$.

40. TADA Y, NAKASE $M$, et al. (1996). Reduction of 14-16 kDa allergenic proteins in transgenic rice plants by antisense genes. FEBS Letters, 391: 341-5.

41. NAKAMURA R, MATSUDA T (1996). Rice allergenic protein and molecular-genetic approach for hypoallergenic rice. Biosci Biotechnol Biochem, 60 : 1215-21.

42. BURKS, A, KING N, et al. (1999). Modification of a major peanut allergen leads to loss of IgE binding. Int Arch Allergy Immunol, 118: 313-4.

43. RABJOHN P, HELM R, et al. (1999). Molecular cloning and epitope analysis of the peanut allergen Ara h 3. J Clin Invest 103: 535-42.

44. MATOBA N, DOYAMA N, et al. (2001). Design and production of genetically modified soybean protein with anti-hypertensive activity by incorporating potent analogue of ovokinin. FEBS Letters, $497: 50-4$.

45. CHAPMAN MD, SMITH AM, VAILES LD, ARRUDA LK, DHANARAJ V, POMÉS A (2000). Recombinant allergens for diagnosis and therapy of allergic disease. J Allergy Clin Immunol, 106 : 409-18.

46. LEVINE M, DOUGAN G (1998). Optimism over vaccines administered via mucosal surfaces. Lancet $351: 1375-6$.

47. DONNET-HUGHES A, DUC N, et al. (2000). Bioactive molecules in milk and their role in health and disease: the role of transforming growth factor-beta. Immunol Cell Biol, 78 : 74-9.

* Texte de la conférence prononcée dans le cadre du Colloque OGM et bénéfices pour la santé organisé par l'Afssa les 17 et 18 décembre 2001 à Paris. 


\section{Illustrations}

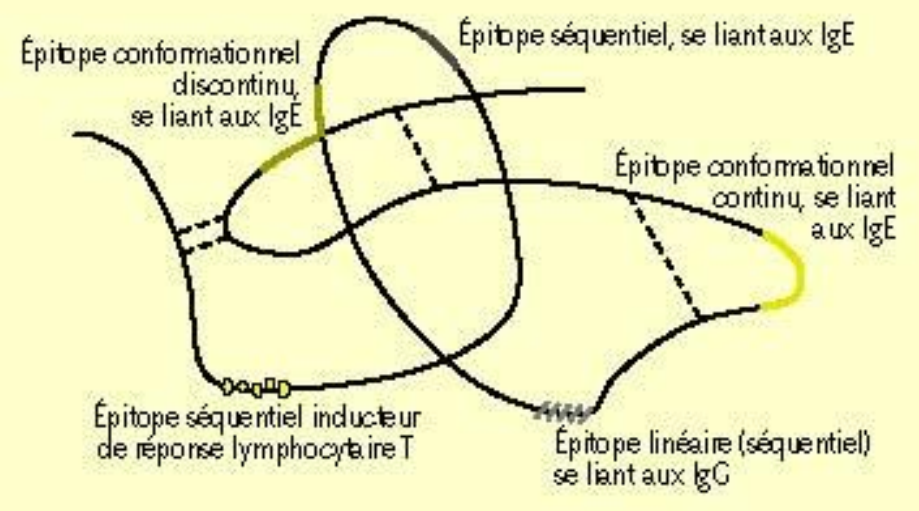

Figure 1. Schéma d'un allergène.

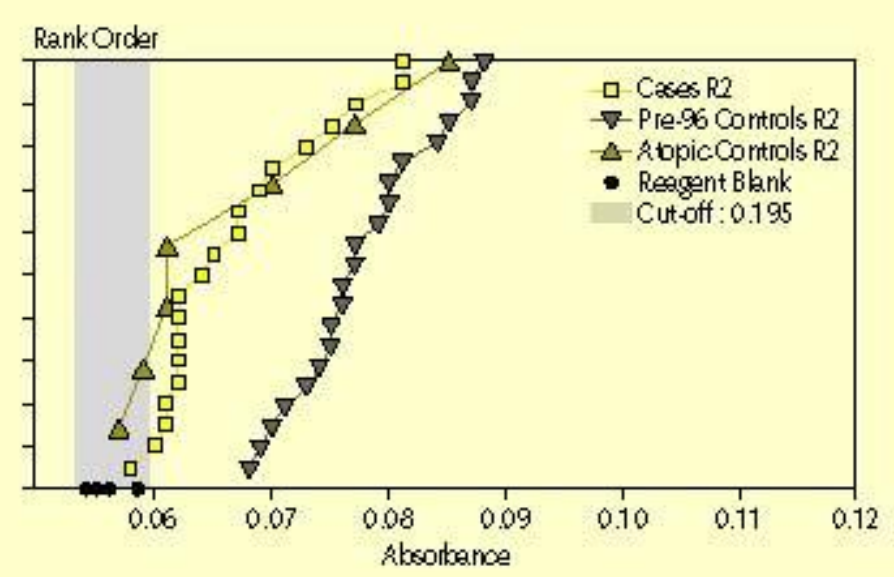

Figure 2. Exemple de la protéine Cry9c (maïs OGM). 